\title{
Discriminação da cobertura vegetal do Cerrado matogrossense por meio de imagens MODIS
}

\author{
Gustavo Bayma Siqueira da Silva(1), Antonio Roberto Formaggio(1), Yosio Edemir Shimabukuro(1), \\ Marcos Adami(1) e Edson Eyji Sano(2) \\ ${ }^{(1)}$ Instituto Nacional de Pesquisas Espaciais, Caixa Postal 515, CEP 12245-970 São José dos Campos, SP. E-mail: bayma@dsr.inpe.br, \\ formag@dsr.inpe.br, yosio@dsr.inpe.br, adami@dsr.inpe.br ${ }^{(2)}$ Embrapa Cerrados, BR 020, Km 18, Caixa Postal 08223, CEP $73310-970$ Planaltina, DF. \\ E-mail: sano@cpac.embrapa.br
}

Resumo - O objetivo do presente trabalho foi avaliar o potencial de uso do modelo linear de mistura espectral (MLME), aplicado em imagens "Moderate Resolution Imaging Spectroradiometer" (MODIS), para discriminar as classes de cobertura vegetal natural e antropogênica do Cerrado matogrossense. O monitoramento do bioma Cerrado está se tornando muito importante devido à sua forte antropização, principalmente nas últimas quatro décadas. Nesse contexto, o sensor MODIS apresenta-se como opção devido à sua alta resolução temporal. Entretanto, considerando sua moderada resolução espacial, é indicada a decomposição de sua resposta espectral. O MLME apresenta-se como uma técnica viável, pois permite estimar o percentual dos componentes do pixel. Os dados utilizados nos perfis temporais das classes corresponderam às seguintes imagens fração do MLME: vegetação, solo e sombra. A discriminação das classes naturais e antropogênicas foi avaliada por meio do cálculo da distância Mahalanobis e apresentada por meio de dendrogramas. As imagens fração permitem análises de séries temporais na caracterização espacial e temporal das classes. As imagens fração solo e sombra, na estação seca, apresentam melhores resultados na discriminação das classes selecionadas. Para discriminação de classes com composições florísticas semelhantes, são indicadas as imagens fração da estação chuvosa.

Termos para indexação: sensoriamento remoto, modelo linear de mistura espectral, análise temporal.

\section{Discrimination of Cerrado vegetation cover in the state of Mato Grosso using MODIS images}

\begin{abstract}
The objective of the present work was to evaluate the potential of the spectral linear mixture model (SLMM), applied to Moderate Resolution Imaging Spectroradiometer (MODIS) images, to discriminate natural and anthropic classes of vegetation in the portion of Mato Grosso state covered by Cerrado vegetation. The monitoring of the Cerrado biome is becoming very important due to its strong human disturbance, especially in the last four decades. In this context, the MODIS sensor appears as an option due to its high temporal resolution. However, considering its moderate spatial resolution, the decomposition of its spectral response is indicated. The SLMM appears to be a viable technique, since it permits estimating the percentage of components within the pixel. The data used in the temporal class profiles corresponded to the following fraction images derived from SLMM: vegetation, soil, and shade. Discrimination of natural and anthropogenic classes was determined through the Mahalanobis distance, presented by dendrograms. The fraction images allow time series analyses for spatial and temporal characterization of the classes. Soil and shade fraction images, in the dry season, present better results in the discrimination of selected classes. For the discrimination of classes with similar floristic composition, fraction images from the rainy season are indicated.
\end{abstract}

Index terms: remote sensing, linear spectral mixture model, temporal analysis.

\section{Introdução}

O bioma Cerrado ocupa uma área de aproximadamente dois milhões de quilômetros quadrados e está localizado na porção central do Brasil. Abrange diferentes paisagens e tipos de vegetação, com um gradiente vegetacional dividido entre formações florestais, savânicas e campestres. Devido à sua diversidade florística, esse bioma está entre os 25 sítios de alta diversidade para conservação da biodiversidade do planeta (Eiten, 1972; Instituto Brasileiro de Geografia e Estatística, 1992; Myers et al., 2000).

Nas últimas décadas, houve grande substituição da vegetação natural do Cerrado por áreas destinadas às atividades agropecuárias (Sano et al., 2002; Sano et al., 2008). As principais atividades passaram a ser

Pesq. agropec. bras., Brasília, v.45, n.2, p.186-194, fev. 2010 
os plantios de soja, de eucalipto e o corte de espécies lenhosas nativas para produção de carvão vegetal. Essas atividades têm afetado a biodiversidade do Cerrado (Furley, 1999). Para melhor análise da dinâmica de antropização desse bioma, é necessário desenvolver e adotar métodos efetivos de avaliação e acompanhamento temporal via detecção de mudanças, para fornecer classificações adequadas do uso e cobertura do solo (Jepson, 2005).

Assim, torna-se necessário um sistema de monitoramento sistemático e operacional para o Cerrado, pois, apesar do ritmo acelerado de conversão, existem poucas tentativas de monitoramento da degradação do bioma. Dados recentes indicam um desmatamento médio anual, entre 2002 e 2008, de $14.200 \mathrm{~km}^{2}$ (B, 2009). Dessa forma, verifica-se crescente necessidade de que esse monitoramento seja realizado respeitando particularidades como o efeito da sazonalidade na vegetação (Sano \& Ferreira, 2005).

Na Amazônia, o monitoramento é feito por meio do Programa de Cálculo do Desflorestamento da Amazônia (PRODES), (Câmara et al., 2006) e pelo Projeto de Detecção de Áreas Desflorestadas em Tempo Real (DETER) (Shimabukuro et al., 2006), com uso de imagens de sensoriamento remoto. A exemplo do DETER, alguns trabalhos têm apontado a capacidade do MODIS em acompanhar e detectar mudanças das classes de uso e cobertura do solo no Cerrado (Galford et al., 2008). Um exemplo de monitoramento para o bioma Cerrado é o Sistema Integrado de Alerta de Desmatamento (SIAD), atualmente desenvolvido para o Estado de Goiás. O SIAD tem como objetivo monitorar sistematicamente o Cerrado goiano, buscando também instrumentos para o controle e redução dos índices de desmatamento (Ferreira et al., 2005).

Nesse sentido, as imagens MODIS e seus produtos têm sido utilizados em trabalhos para o estudo da fenologia da cobertura vegetal (Ferreira Júnior et al., 2003; Ferreira Júnior \& Huete, 2004; Freitas \& Shimabukuro, 2008), monitoramento da dinâmica espaço-temporal de biomas (Adami et al., 2008) e para a detecção das mudanças ocorridas no uso e na cobertura do solo (Galford et al., 2008; Hayes et al., 2008; Epiphanio et al., 2010).

Entretanto, como a melhor resolução espacial do sensor MODIS é de $250 \mathrm{~m}$, a resposta em um pixel é resultante da integração das radiâncias de diferentes alvos, e ocasiona a predominância de mistura espectral intra-pixel. Assim, o emprego de um procedimento que permita decompor essa resposta foi indicado por Aguiar et al. (1999) e Carvalho Júnior et al. (2003). Para essa decomposição, com estimativa do percentual correspondente de cada componente, Shimabukuro \& Smith (1991) propuseram o uso do modelo linear de mistura espectral (MLME), que visa gerar imagens sintéticas (imagens fração) que representem as proporções de cada componente na formação do pixel. Todavia, mais pesquisas são necessárias a fim de avaliar o desempenho do MLME no mapeamento e monitoramento do Cerrado (Ferreira et al., 2007).

Dessa forma, o objetivo do presente trabalho foi avaliar o potencial do MLME aplicado a imagens MODIS para discriminar as classes de vegetação natural e antropogênica do Cerrado matogrossense.

\section{Material e Métodos}

A área de estudo corresponde à porção do bioma Cerrado encontrada no Estado do Mato Grosso, entre as latitudes $09^{\circ} 45^{\prime} \mathrm{S}$ e $18^{\circ} 10^{\prime} \mathrm{S}$ e as longitudes $50^{\circ} 00^{\prime} \mathrm{W}$ e $60^{\circ} 25^{\prime} \mathrm{W}$ (Figura 1). Nessa área, há grande pressão antrópica resultante da acentuada conversão da vegetação natural em agricultura ou pastagem, decorrente do incentivo do Governo Federal no início da década de 1970, que impulsionou a expansão da agricultura no Centro-Oeste brasileiro.

A caracterização sazonal da precipitação na área de estudo foi obtida utilizando dados do satélite TRMM (Tropical Rainforest Measuring Mission). Os dados de precipitação entre as latitudes $35^{\circ} \mathrm{N}$ e $35^{\circ} \mathrm{S}$ são coletados desde 1997 . Esse satélite auxilia a

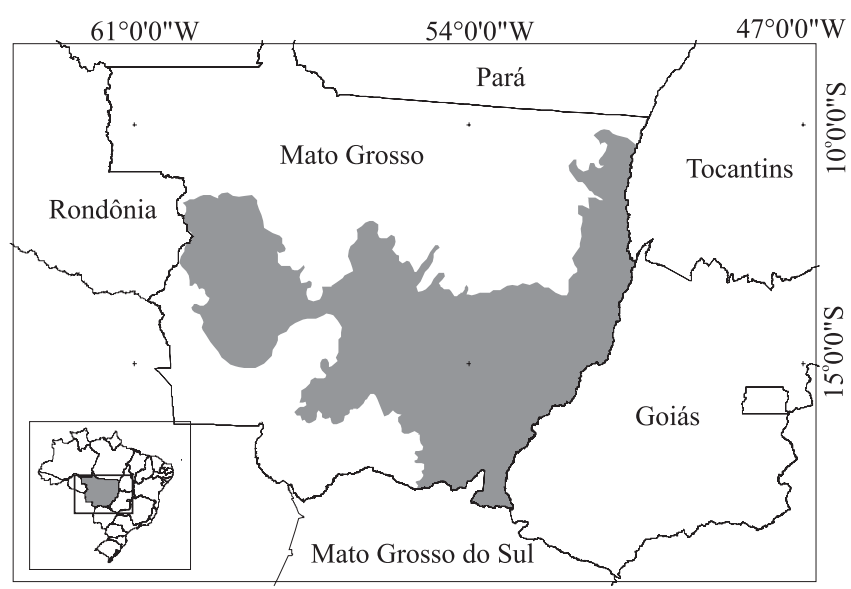

Figura 1. Porção do estado de Mato Grosso coberta pelo bioma Cerrado. 
estimação de precipitação, uma vez que as estimativas convencionais de precipitação nos trópicos são normalmente feitas com base em modelos de previsão climática que utilizam dados obtidos por radares terrestres que cobrem parcelas de áreas menores que as cobertas pelo TRMM (Kummerow et al., 2000). Os dados utilizados neste trabalho referem-se aos produtos 3B43, com resolução espacial em torno de $30 \mathrm{~km}$, e estão disponíveis na internet (http://mirador. gsfc.nasa.gov>). A caracterização da precipitação na área de estudo é apresentada na Figura 2.

No bioma Cerrado, três categorias de formação vegetal devem ser consideradas: florestais, savânicas e campestres. No presente estudo, somente foram consideradas as savanas, que correspondem a uma vegetação xeromorfa, de clima estacional, que pode ser encontrada em clima ombrófilo, e reveste solos lixiviados ou aluminizados (Instituto Brasileiro de Geografia e Estatística, 1992). São encontrados, na área de estudo, quatro subgrupos: savana gramíneo-lenhosa $(\mathrm{Sg})$, onde prevalecem os gramados entremeados por plantas lenhosas raquíticas; savana parque (Sp), constituída essencialmente por um estrato de gramíneas integrado com um estrato arbustivo; savana arborizada $(\mathrm{Sa})$, caracterizada por apresentar fisionomia monofanerofítica rala e hemicriptofítica graminoide contínua; e savana florestada (Sd), restrita a áreas areníticas lixiviadas com solos profundos e com plantas de estrutura lenhosa.
Até o ano 2002, dos $382.170 \mathrm{~km}^{2}$ de extensão da área de estudo, 22,8\% correspondiam às áreas de $\mathrm{Sa}, 21,1 \%$ correspondiam à classe $\mathrm{Sp}, 18,3 \%$ correspondiam às áreas de pastagem cultivadas (Ap), 15,3\% correspondiam a culturas agrícolas (Ac), $8 \%$ correspondiam à área de $\mathrm{Sd}, \quad 0,3 \%$ correspondiam à classe de $\mathrm{Sg}$ e 14,2\% correspondiam a outras classes (áreas de floresta, corpos d'água etc.) (Brasil, 2007).

A seleção das amostras das classes de vegetação natural e antropogênica foi baseada no mapa de áreas desmatadas do Cerrado matogrossense de 2001 (Silva, 2009) e no mapa de cobertura vegetal que discrimina as classes naturais e antropogênicas de Cerrado no ano de 2002 (Brasil, 2007). A intersecção desses dois mapas permitiu localizar as áreas sem alteração do uso e da cobertura da terra. As amostras foram selecionadas nos centroides dos polígonos, para evitar influência de bordas. Com base no resultado da intersecção, foram selecionados seis conjuntos de amostras, um por classe, a saber: $\mathrm{Sd}, \mathrm{Sa}, \mathrm{Sp}, \mathrm{Sg}$, Ap e Ac. Cada conjunto foi composto por 100 amostras.

Para a elaboração dos perfis temporais, foram utilizadas 46 imagens (outubro de 2000 a outubro de 2002) do produto MOD13 (índices de vegetação) do sensor MODIS, na resolução espacial de $250 \mathrm{~m}$, com composição de 16 dias, referentes à camada h12v10 (Soares at al., 2007). Nesses dados MODIS, foi aplicado o MLME:

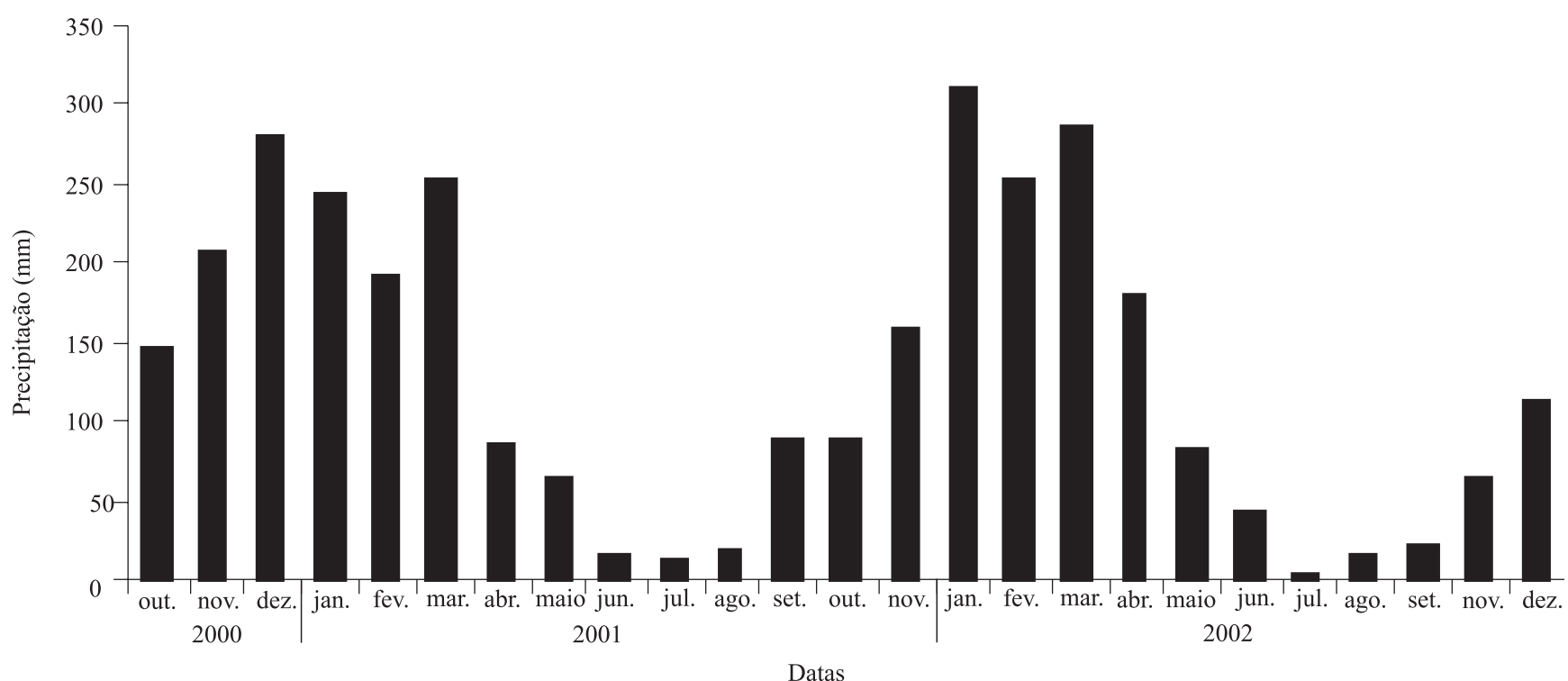

Figura 2. Precipitação média mensal na área de estudo, entre outubro de 2000 e outubro de 2002, estimada pelo sensor orbital "Tropical Rainfall Measuring Mission". 
$\mathrm{r}_{\mathrm{i}}=\mathrm{a} \mathrm{veg}_{\mathrm{i}}+\mathrm{b} \mathrm{solo}_{\mathrm{i}}+\mathrm{c} \mathrm{sombra}_{\mathrm{i}}+\mathrm{e}_{\mathrm{i}}$, em que $\mathrm{r}_{\mathrm{i}}$ é a resposta do pixel na banda i da imagem; a, b e c são proporções de vegetação, solo e sombra ou água que compõem o pixel; veg, solo e sombra correspondem às respostas espectrais de cada um desses componentes; e e é o erro de estimação intrínseco para cada banda i. Deve ser considerada a restrição dos componentes, em que não podem existir resultados inferiores a zero e o somatório de suas proporções tem que ser igual a um (Shimabukuro \& Smith, 1991; Aguiar et al., 1999).

Para decompor as imagens pelo MLME, é necessária a seleção de pixels puros que representem cada um dos componentes. Essa seleção foi realizada usando duas imagens MOD13 das estações seca e chuvosa. Na imagem da estação seca, foram obtidos os valores de reflectância para a seleção do pixel puro de solo (julho de 2002), pois nessa estação a probabilidade de encontrar áreas com solo exposto é maior. Na imagem da estação chuvosa (fevereiro de 2002), foram selecionados os pixels puros de vegetação (devido ao maior vigor vegetativo nessa estação) e de sombra. Os valores de reflectância dos pixels puros selecionados (Figura 3) foram utilizados pelo MLME e aplicados nas bandas do vermelho (620 a $670 \mathrm{~nm}$ ), infravermelho próximo (841 a $876 \mathrm{~nm})$, azul (459 a $479 \mathrm{~nm})$ e infravermelho médio (2.105 a $2.155 \mathrm{~nm}$ ) da série temporal utilizada.

Os perfis temporais dos componentes foram analisados graficamente. Foi aplicado o teste Kolmogorov-Smirnov para verificar se havia diferença significativa entre os resultados de cada classe, para cada mês, em cada componente. O teste de Kolmogorov-Smirnov foi utilizado porque, após a aplicação do teste de Lilleford, não foi observada normalidade dos dados. As diferenças significativas

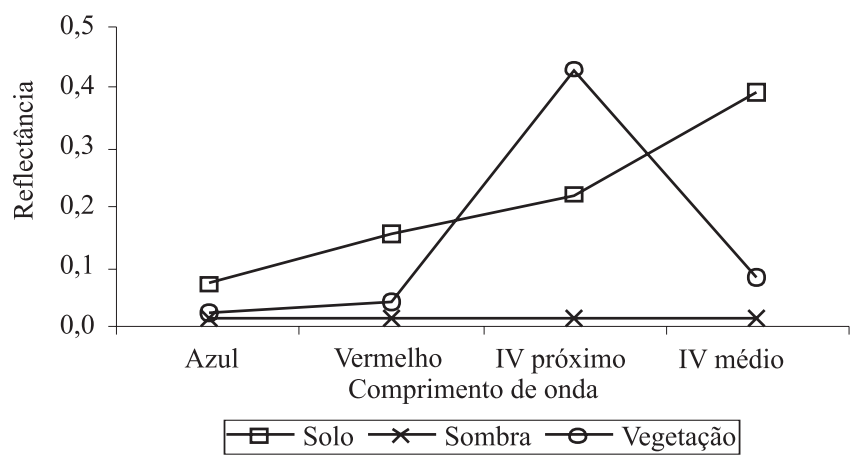

Figura 3. Curvas espectrais dos pixels puros de solo, sombra e vegetação. $(\mathrm{p}<0,05)$ foram agrupadas, e também foi apresentado o número de datas em que foi possível a discriminação dos conjuntos amostrais das classes de interesse. Esses dados também foram agrupados para as duas estações, seca e chuvosa, em função da possibilidade de obtenção de imagens livres de nuvens (Sano et al., 2007).

Para verificar a possibilidade de discriminar as classes, foi calculada a distância Mahalanobis entre as médias de cada conjunto amostral. A apresentação das distâncias entre as médias dos conjuntos amostrais foi realizada por meio de dendrogramas, utilizando o método de Ward para formar os agrupamentos. Esse método forma os agrupamentos minimizando a dissimilaridade (minimizando o total das somas de quadrados dos desvios) entre dois grupos ( $\mathrm{R} \mathrm{e} \mathrm{S}$ ), apresentada na equação

$$
\mathrm{d}_{(\mathrm{r}, \mathrm{s})}^{2}=\mathrm{n}_{\mathrm{r}} \mathrm{n}_{\mathrm{s}}\left[\left(\mathrm{x}_{\mathrm{r}}-\mathrm{x}_{\mathrm{s}}\right) \mathrm{V}^{-1}\left(\mathrm{x}_{\mathrm{r}}-\mathrm{x}_{\mathrm{s}}\right)^{\prime}\right]^{2 /} /\left(\mathrm{n}_{\mathrm{r}}+\mathrm{n}_{\mathrm{s}}\right),
$$

em que: $\mathrm{n}_{\mathrm{r}}$ e $\mathrm{n}_{\mathrm{s}}$ representam o conjunto de dados em cada agrupamento; $\mathrm{x}_{\mathrm{r}}$ e $\mathrm{x}_{\mathrm{s}}$ representam a matriz de elementos de cada agrupamento; $V$ é a matriz de covariância entre os agrupamentos. Em cada etapa do procedimento são formados grupos, de tal maneira que a solução resultante tenha a menor soma dos quadrados dos desvios dentro dos grupos. Nessas etapas, são consideradas as uniões de todos os possíveis pares de grupos, e os dois que resultam em menor aumento da soma dos quadrados dos desvios são agrupados até que todos formem um único grupo, reunindo todos os indivíduos. Dessa maneira, o método de Ward diminui o efeito de "outliers" na análise, formando grupos com menor variância intra-agrupamentos e maior variância intergrupos (Mingoti, 2005).

\section{Resultados e Discussão}

Na Figura 4 A, são apresentados os perfis médios do componente solo. Os retângulos tracejados indicam o período de seca, de maio a setembro, conforme Radambrasil (1982) e dados TRMM apresentados na Figura 2. Os picos dos valores das proporções corresponderam ao fim do período de seca. Também nesse período, as curvas apresentaram-se mais espaçadas. Esse resultado corrobora os resultados obtidos por Ferreira Júnior \& Huete (2004) e Liesenberg et al. (2007b), em que o final do período de seca também foi o de maior separabilidade entre as classes de Cerrado. 
Na Figura 4 B, são apresentados os perfis médios da sombra. Esse componente apresentou maiores ruídos, principalmente na estação chuvosa, com intervalos maiores que no componente solo. Isso pode ser decorrente da presença de sombras de nuvens, que é intensa nessa estação. Em relação à vegetação (Figura $4 \mathrm{C}$ ), destaca-se a classe Ac, com picos na estação chuvosa. Esse
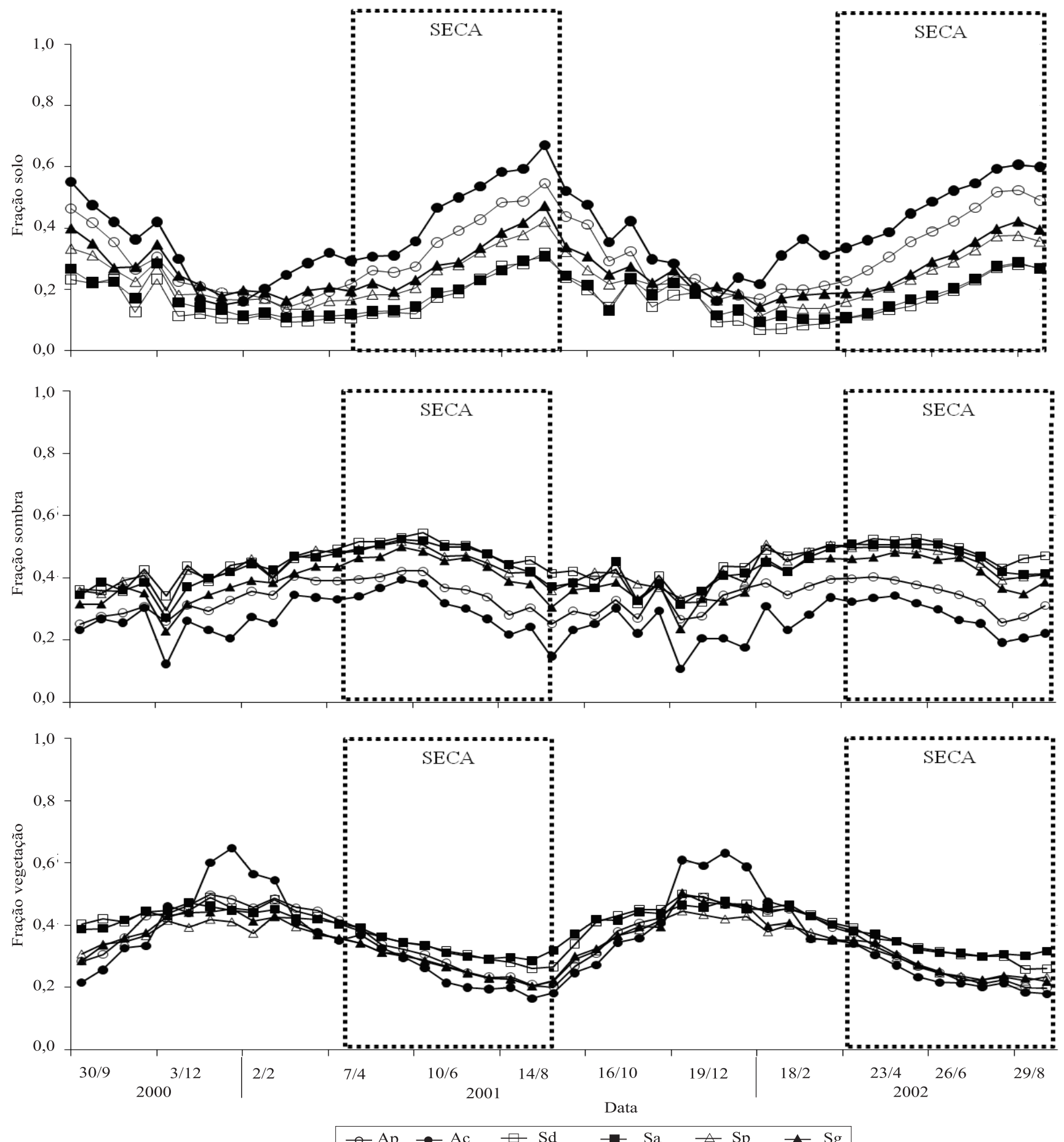

Figura 4. Perfis temporais das classes de vegetação de Cerrado e de uso antropogênico nos componentes solo (A), sombra (B) e vegetação (C). Ac, culturas agrícolas; Ap, pastagens cultivadas; Sd, savana arborizada; Sp, savana parque; Sg, savana gramíneo-lenhosa. 
período coincide com o período de maior vigor da maioria das culturas anuais na área de estudo, como a soja. Em função desse comportamento, o componente vegetação pode ser indicado para a discriminação de áreas de culturas agrícolas, na estação chuvosa.

A variabilidade dos valores dos componentes em relação às classes de Cerrado ocorreu em função do porte, da estrutura, do vigor da vegetação e da época do ano. A maior variação para as classes $\mathrm{Sp}$ e $\mathrm{Sg}$ no solo e na vegetação pode ser explicada pela perda mais acentuada do vigor e de folhas na estação seca do que a ocorrida nas classes Sd e Sa. Observou-se que os picos dos valores do solo correspondem às mínimas de precipitação média. O componente vegetação mostra que o pico da classe Ac ocorre também na máxima de precipitação média, em ambos os anos considerados. Podem ser percebidas amplitudes maiores nos perfis referentes às classes antropogênicas (Ac e Ap), principalmente no solo e na vegetação. Entre as classes de vegetação natural de Cerrado, o comportamento do solo foi coerente com a estrutura da vegetação, pois a estrutura do dossel em formações florestais é mais densa, e acarreta menores valores dessa proporção do que entre as classes savânicas do Cerrado (Figura 4 A, B e C.)

$\mathrm{Na}$ Figura $5 \mathrm{~A}$, correspondente ao dendrograma do solo, pode ser observado o agrupamento das classes de vegetação natural e antropogênica. Neste componente, foram detectadas as diferenças na estrutura da vegetação, pois as classes com predominância arbórea e arbustiva ( $\mathrm{Sd}$ e $\mathrm{Sa}$ ), com dosséis mais fechados, apresentaram-se separadas daquelas com predominância de gramíneas ( $\mathrm{Sp}$ e $\mathrm{Sg}$ ) e das de uso antrópico (Ap e Ac). A distância entre o agrupamento $\mathrm{Sd}$ e $\mathrm{Sa}$ foi menor do que a distância entre o agrupamento $\mathrm{Sp}$ e $\mathrm{Sg}$, o que de certa forma era esperado em razão da classe $\mathrm{Sp}$ apresentar vegetação também do tipo arbustivo e a classe $\mathrm{Sg}$ apresentar ausência quase completa desse tipo de vegetação. Ainda assim, essas classes foram discriminadas das classes $\mathrm{Sd}$ e Sa, que apresentaram maior densidade vegetacional.

A Figura 5 B corresponde aos dados da sombra. Esse componente ressalta a diferença entre as classes de cobertura vegetal natural de Cerrado e cobertura vegetal antropogênica. Entre as classes de vegetação de Cerrado, a Sg apresentou-se separada das demais. Isso de certa forma era esperado, já que, entre as selecionadas, essa é a única classe que apresenta predominância de estrato graminoso, com ausência quase completa do estrato arbóreo-arbustivo. Mesmo assim, a distância entre a média dessa classe e a média da classe de uso antrópico (Ap) permitiu diferenciar a pastagem natural da pastagem cultivada. Em dados de vegetação obtidos por sensoriamento remoto, deve ser considerado o efeito de fundo ocasionado principalmente pela resposta do solo. Dependendo desse efeito, a resposta espectral do alvo é alterada. Isso acaba por possibilitar a discriminação, por exemplo, entre formas diferentes de manejo e níveis de degradação. Assim, o efeito de fundo do substrato e do solo pode ser condicionado pela biomassa da vegetação, que varia em função da intensidade diferenciada de pastoreio, da homogeneidade e da densidade vegetacional.

$\mathrm{Na}$ Figura $5 \mathrm{C}$, é apresentado o dendrograma da vegetação, no qual pode-se observar que as classes apresentam menores distâncias entre si, quando comparadas aos componentes solo e sombra. A maior distância observada nesse componente apresentou valor próximo a oito, enquanto, para solo e sombra, as maiores distâncias foram próximas a 12 . Os agrupamentos das classes, nesse componente, foram coerentes com as características das classes de vegetação, uma vez que foram agrupadas $\mathrm{Sp}, \mathrm{Sg}$ e Ap com dosséis mais esparsos, e Sa e Sd com dosséis mais fechados. Esse componente permite separar a classe Ac das demais. Cabe ressaltar também que a classe Ac apresentou-se separada das demais nos componentes solo e vegetação, e a classe Ap não foi separada das classes de vegetação natural apenas no componente vegetação.

O resultado do teste Kolmogorov-Smirnov permitiu avaliar a discriminação das classes em cada data. Esse resultado foi agrupado para as estações seca, chuvosa e em todo o período. O período de seca correspondeu a 20 das 46 datas analisadas (Figura 6 A). No conjunto de dados desse período, o solo e sombra permitiram maior discriminação das classes do que a vegetação. As discriminações entre $\mathrm{Sa}$ e $\mathrm{Sd}$ e entre $\mathrm{Sp}$ e $\mathrm{Sg}$ não foram tão eficientes, com diferença significativa em menos da metade das datas, pois, na estação seca, as classes com composição florística semelhante apresentaram maior dificuldade de discriminação. Os dados do MLME foram eficientes na discriminação das classes natural e antropogênica, pois pelo menos 15 datas apresentaram diferenças significativas em pelo menos um dos componentes.

A estação chuvosa apresentou 26 datas das 46 correspondentes à série temporal. $\mathrm{O}$ desempenho 
na discriminação do conjunto de dados das classes na estação chuvosa foi um pouco inferior ao da estação seca (Figura 6 B). Entretanto, para o conjunto de classes que apresentaram dificuldade de discriminação no período seco (Sa-Sd e $\mathrm{Sp}-\mathrm{Sg}$ ), a estação chuvosa apresentou melhores resultados. Isso pode ser explicado pelo maior vigor da vegetação na estação chuvosa, com toda a estrutura de caules e folhas desenvolvidas em sua plenitude. Na época seca, a vegetação perde parte da estrutura foliar e lenhosa, mascarando a resposta
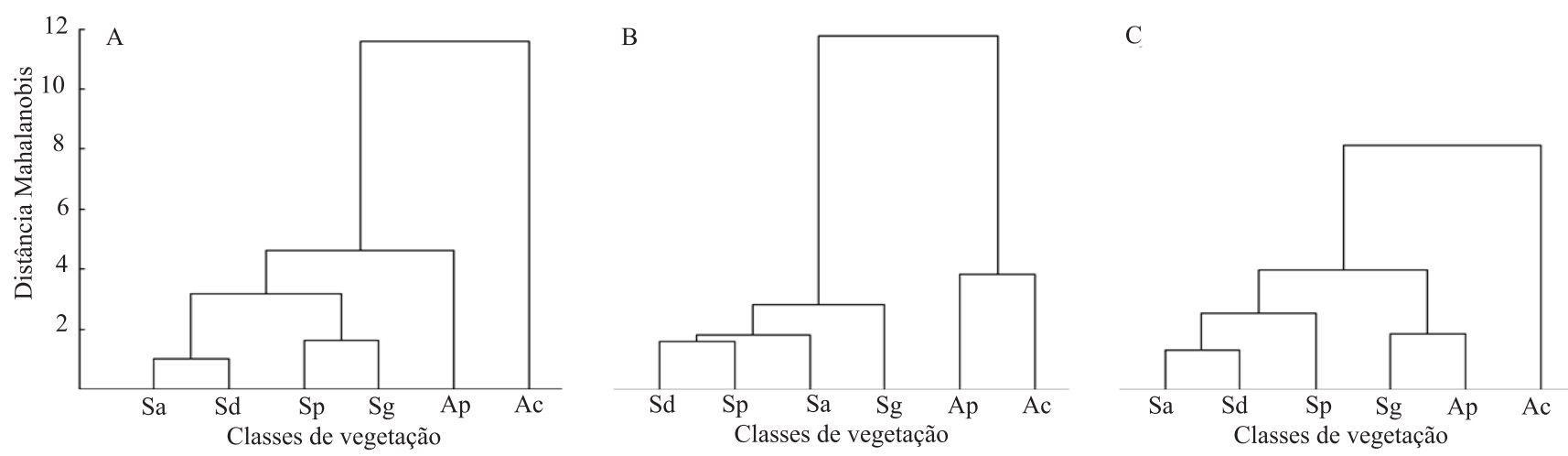

Figura 5. Dendrogramas com as distâncias entre as classes de vegetação e uso antropogênico nos componentes solo (A), sombra (B) e vegetação (C). Ac, culturas agrícolas; Ap, pastagens cultivadas; Sd, savana arborizada; Sp, savana parque; Sg, savana gramíneo-lenhosa.
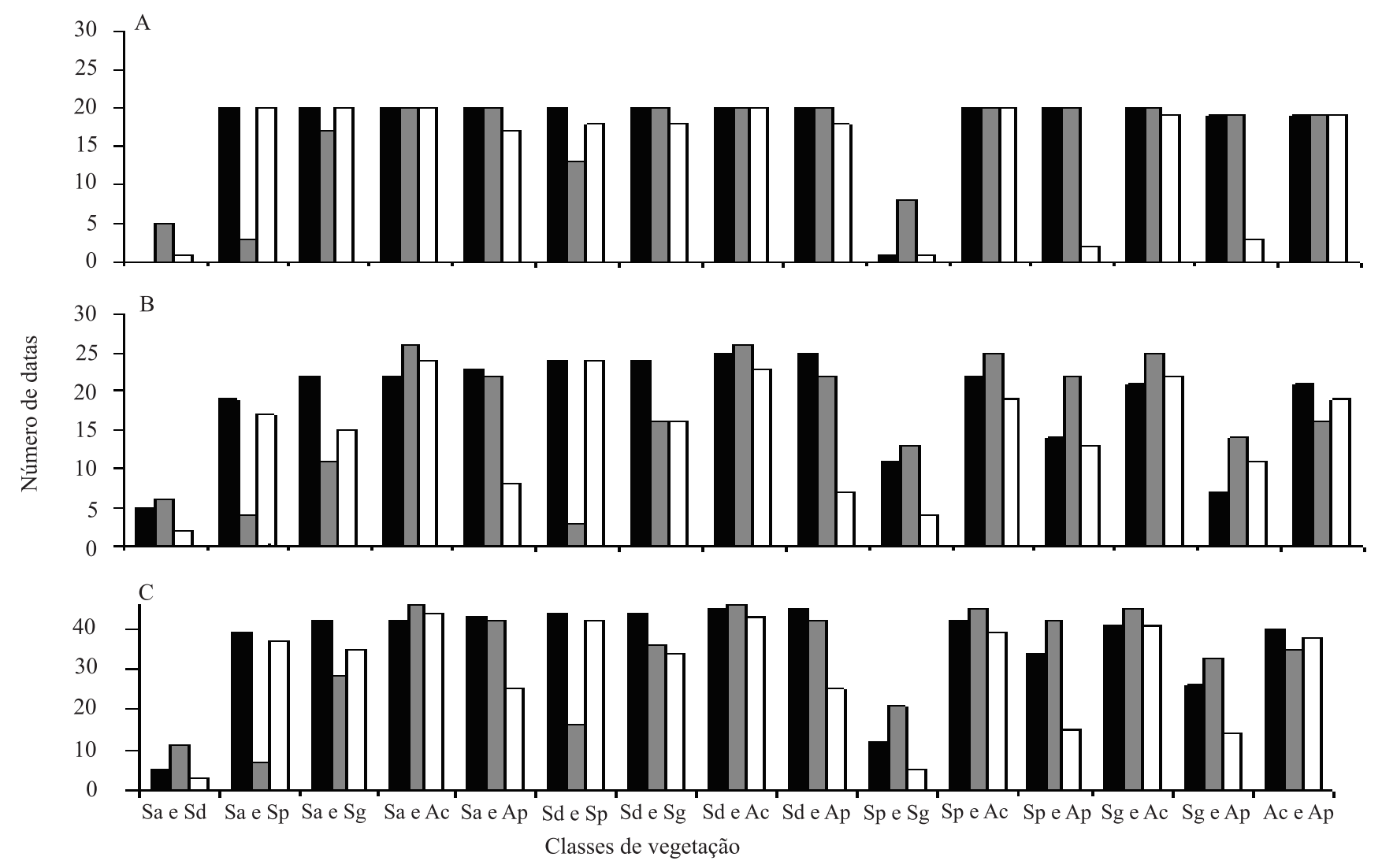

$\square$ Solo $\square$ Sombra $\square$ Vegetação

Figura 6. Número de ocorrências de datas com diferença significativa entre as classes na estação seca (A), chuvosa (B) e em todas as datas (C), para os componentes solo, sombra e vegetação. 
espectral e confundindo classes de composição florística semelhantes.

Analisando os resultados entre as classes, por estação do ano, pode ser observada a influência da sazonalidade no comportamento das classes de vegetação natural e antropogênica, com diferenças significativas maiores entre as classes na época seca do que na época de chuva. Isso corrobora o trabalho de Liesenberg et al. (2007a), que observaram comportamento semelhante ao discriminar classes de vegetação de Cerrado com dados do sensor "multi-angle imaging spectroradiometer" (MISR).

O resultado global dessa avaliação é apresentado na Figura 6 C. A discriminação entre as classes de Cerrado natural e as áreas antropogênicas (Ac e Ap) ocorreu principalmente nos componentes solo e sombra, para a maioria das datas. Isso pode ser explicado pela relação entre esses componentes e a estrutura vegetacional das classes. A discriminação foi menos eficiente para o conjunto de dados das classes $\mathrm{Sa}-\mathrm{Sd}$ e $\mathrm{Sp}-\mathrm{Sg}$, com menos da metade das datas apresentando diferenças significativas, para os três componentes. Isso já era esperado, já que as duas classes de vegetação que compõem cada conjunto apresentam composição florística semelhante.

Os resultados deste estudo indicaram que as imagens fração originadas do MLME são viáveis e práticas, na caracterização de formações savânicas e florestais e classes de uso antropogênicas (pastagens cultivadas/ culturas agrícolas), e corroboram o trabalho de Ferreira et al. (2007).

\section{Conclusões}

1. As imagens fração derivadas de MLME e de dados MODIS permitem discriminar classes de cobertura vegetal natural e antropogênica do Cerrado.

2. As imagens fração solo e sombra, da estação seca, apresentam melhores resultados na discriminação das classes de cobertura vegetal selecionadas neste estudo.

3. Para discriminação de classes de cobertura vegetal natural com composições florísticas semelhantes, são indicadas as imagens fração da estação chuvosa.

\section{Agradecimentos}

À Coordenação de Aperfeiçoamento de Pessoal de Nível Superior, pela bolsa concedida para o desenvolvimento do presente trabalho.

\section{Referências}

ADAMI, M.; FREITAS, R.M. de; PADOVANI, C.R.; SHIMABUKURO, Y.E.; MOREIRA, M.A. Estudo da dinâmica espaço-temporal do bioma Pantanal por meio de imagens MODIS. Pesquisa Agropecuária Brasileira, v.43, p.1371-1378, 2008.

AGUIAR, A.P.D.; SHIMABUKURO, Y.E.; MASCARENHAS, N.D.A. Use of synthetic bands derived from mixing models in the multispectral classification of remote sensing images. International Journal of Remote Sensing, v.20, p.647-657, 1999.

BRASIL. Ministério do Meio Ambiente. Mapeamento de cobertura vegetal do bioma Cerrado: relatório final. Brasília, 2007. 93p.

BRASIL. Ministério do Meio Ambiente. Relatório técnico de monitoramento do desmatamento no bioma Cerrado, 2002 a 2008: dados revisados. Brasília: MMA, 2009. 67p. Disponível em: $<$ http://www.mma.gov.br/estruturas/sbf_chm_rbbio/_arquivos/ relatorio_tecnico_monitoramento_desmate_bioma_cerrado_csr ibama_2002_2008_rev_72.pdf $>$. Acesso em: 10 mar. 2010.

CÂMARA, G.; VALERIANO, D.M.; SOARES, J.V. Metodologia para o cálculo da taxa anual de desmatamento na Amazônia Legal. São José dos Campos: Inpe, 2006. 24p. Disponível em: $<$ http://www.obt.inpe.br/prodes/ index.html $>$. Acesso em: 10 maio 2008.

CARVALHO JÚNIOR, O.A.; CARVALHO, A.P.F.D.; GUIMARÃES, R.F.; MENESES, P.R. Mistura espectral: (I) detecção dos membros finais utilizando a geometria do simplex. Espaço \& Geografia, v.6, p.147-173, 2003.

EITEN, G. The Cerrado vegetation of Brazil. The Botanical Review, v.38, p.201-341, 1972.

EPIPHANIO, R.D.V.; FORMAGGIO, A.R.; RUDORFF, B.F.T.; MAEDA, E.E.; LUIZ, A.J.B. Estimating soybean crop areas using spectral-temporal surfaces derived from MODIS images in Mato Grosso, Brazil. Pesquisa Agropecuária Brasileira, v.45, p.72-80, 2010.

FERREIRA, M.E.; FERREIRA JÚNIOR, L.G.; FERREIRA, N.C. (Coord.). Sistema Integrado de Alerta de Desmatamento para o Estado de Goiás-SIAD. Goiânia: UFG, 2005. 72p.

FERREIRA, M.E.; FERREIRA JÚNIOR, L.G.; SANO, E.E.; SHIMABUKURO, Y.E. Spectral linear mixture modelling approaches for land cover mapping of tropical savanna areas in Brazil. International Journal of Remote Sensing, v.28, p.413429, 2007.

FERREIRA JÚNIOR, L.G.; HUETE, A.R. Assessing the seasonal dynamics of the Brazilian Cerrado vegetation through the use of spectral vegetation indices. International Journal of Remote Sensing, v.25, p.1837-1860, 2004.

FERREIRA JÚNIOR, L.G.; YOSHIOKA, H.; HUETE, A.R.; SANO, E.E. Seasonal landscape and spectral vegetation index 
dynamics in the Brazilian Cerrado: an analysis within the Large-Scale Biosphere-Atmosphere experiment in Amazônia (LBA). Remote Sensing of Environment, v.87, p.534-550, 2003.

FREITAS, R.M.; SHIMABUKURO, Y.E. Combining wavelets and linear spectral mixture model for MODIS satellite sensor timeseries analysis. Journal of Computational Interdisciplinary Sciences, v.1, p.33-38, 2008.

FURLEY, P.A. The nature and diversity of neotropical savanna vegetation with particular reference to the Brazilian Cerrados. Global Ecology and Biogeography, v.8, p.223-241, 1999.

GALFORD, G.L.; MUSTARD, J.F.; MELILLO, J.; GENDRIN, A.; CERRI; C.C.; CERRI, C.E.P. Wavelet analysis of MODIS time series to detect expansion and intensification of row-crop agriculture in Brazil. Remote Sensing of Environment, v.112, p.576-587, 2008.

HAYES, D.J.; COHEN, W.B.; SADER, S.A.; IRWIN, D.E. Estimating proportional change in forest cover as a continuous variable from multi-year MODIS data. Remote Sensing of Environment, v.112, p.735-749, 2008.

INSTITUTO BRASILEIRO DE GEOGRAFIA E ESTATÍSTICA. Manual Técnico da Vegetação Brasileira. Rio de Janeiro: IBGE, 1992.

JEPSON, W.A disappearing biome? Reconsidering land-cover change in the Brazilian savanna. The Geographical Journal, v.171, p.99-111, 2005.

KUMMEROW, C.; SIMPSON, J.; THIELE, O.; BARNES, W.; CHANG, A.T.C.; STOCKER, E.; ADLER, R.F.; HOU, A.; KAKAR, R.; WENTZ, F.; ASHCROFT, P.; KOZU, T.; HONG, Y.; OKAMOTO, K.; IGUCHI, T.; KUROIWA, H.; IM, E.; HADDAD, Z.; HUFFMAN, G. The status of the Tropical Rainfall Measuring Mission (TRMM) after two years in orbit. Journal of Applied Meteorology, v.39, p.1965-1982, 2000.

LIESENBERG, V.; GALVÃO, L.S.; PONZONI, F.J. Variations in reflectance with seasonality and viewing geometry: implications for classification of Brazilian savanna physiognomies with MISR/TERRA data. Remote Sensing of Environment, v.107, p.276-286, 2007a.

LIESENBERG, V.; PONZONI, F.J.; GALVÃO, L.S. Análise da dinâmica sazonal e separabilidade espectral de algumas fitofisionomias do Cerrado com índices de vegetação dos sensores Modis/Terra e Aqua. Revista Árvore, v.31, p.295-305, 2007 b.
MINGOTI, S.A. Análise de dados através de métodos de estatística multivariada. Belo Horizonte: UFMG, 2005. 297p.

MYERS, N.; MITTERMEYER, R.A.; MITTERMEYER, C.G.; FONSECA, G.A.; KENT, J. Biodiversity hotspots for conservation priorities. Nature, v.403, p.853-858, 2000.

RADAMBRASIL. Departamento Nacional de Produção Mineral. Folha SD 21: Cuiabá. geologia, geomorfologia, pedologia, vegetação e uso potencial da terra. Rio de Janeiro: DNPM, 1982. 540p. (Levantamento dos Recursos Naturais, 26).

SANO, E.E.; BARCELLOS, A.O.; BEZERRA, H.S. Assessing the spatial distribution of cultivated pastures in the Brazilian savanna. Pasturas Tropicales, v.22, p.2-15, 2002.

SANO, E.E.; FERREIRA, L.G. Monitoramento semidetalhado (escala de 1:250.000) de ocupação de solos do Cerrado: considerações e proposta metodológica. In: SIMPÓSIO BRASILEIRO DE SENSORIAMENTO REMOTO, 12., 2005, Goiânia. Anais. São José dos Campos: Inpe, 2005. p.3309-3316.

SANO, E.E.; FERREIRA, L.G.; ASNER, G.P.; STEINKE, E.T. Spatial and temporal probabilities of obtaining cloud-free Landsat images over the Brazilian tropical savanna. International Journal of Remote Sensing, v.28, p.2739-2752, 2007.

SANO, E.E.; ROSA, R.; BRITO, J.L.S.; FERREIRA, L.G. Mapeamento semidetalhado do uso da terra do Bioma Cerrado. Pesquisa Agropecuária Brasileira, v.43, p.153-156, 2008.

SHIMABUKURO, Y.E.; SMITH, J.A. The least-squares mixing models to generate fraction images derived from remote sensing multispectral data. IEEE Transactions on Geoscience and Remote Sensing, v.29, p.16-20, 1991.

SHIMABUKURO, Y.E.; DUARTE, V.; ANDERSON, L.O.; VALERIANO, D.M.; ARAI, E.; FREITAS, R.M. Near real time detection of deforestation in the Brazilian Amazon using MODIS imagery. Revista Ambi-Água, v.1, p.37-47, 2006.

SILVA, G.B.S. Dados MODIS para deteç̧ão de alterações antrópicas no Cerrado matogrossense. 2009. 113p. Tese (Mestrado) - Instituto Nacional de Pesquisas Espaciais, São José dos Campos.

SOARES, J.V.; BATISTA, G.T.; SHIMABUKURO, Y.E. Histórico e Descrição. In: RUDORFF, B.F.T.; SHIMABUKURO, Y.E.; CEBALLOS, J.C. (Org.). O Sensor MODIS e suas aplicações no Brasil. São José dos Campos: Parêntese, 2007. p.1-22.

$\overline{\text { Recebido em } 28 \text { de outubro de } 2009 \text { e aprovado em } 4 \text { de janeiro de } 2010}$ 